\title{
A review of mirvetuximab soravtansine in the treatment of platinum-resistant ovarian cancer
}

\author{
Kathleen N Moore*, ${ }^{*}$, Lainie P Martin², David M O’Malley³, Ursula A Matulonis ${ }^{4}$, Jason A \\ Konner $^{5}$, Ignace Vergote ${ }^{6}$, Jose F Ponte ${ }^{7}$ \& Michael J Birrer ${ }^{8}$ \\ ${ }^{1}$ Department of Obstetrics \& Gynecology, Stephenson Oklahoma Cancer Center at the University of Oklahoma Health Sciences \\ Center, Oklahoma City, OK 73104, USA \\ 2Department of Hematology/Oncology, Fox Chase Cancer Center, Philadelphia, PA 19111, USA \\ ${ }^{3}$ Department of Obstetrics \& Gynecology, The Ohio State University, Columbus, OH 43210, USA \\ ${ }^{4}$ Department of Medical Oncology, Dana Farber Cancer Institute, Boston, MA 02115, USA \\ ${ }^{5}$ Medical Oncology, Memorial Sloan Kettering Cancer Center, New York, NY 10065, USA \\ ${ }^{6}$ Gynaecological Oncology, Leuven Cancer Institute, Leuven 3000, Belgium \\ ${ }^{7}$ Pharmacology, ImmunoGen, Inc, Waltham, MA 02451, USA \\ ${ }^{8}$ Gillette Center for Gynecologic Oncology, Massachusetts General Hospital, Boston, MA 02114, USA \\ * Author for correspondence: kathleen-moore@ouhsc.edu
}

Resistance to platinum-based therapy poses a significant clinical challenge for the management of advanced ovarian cancer, a leading cause of cancer mortality among women. Mirvetuximab soravtansine is a novel antibody-drug conjugate that targets folate receptor- $\alpha$, a validated molecular target for therapeutic intervention in this disease. Here, we examine mirvetuximab soravtansine's mechanism of action and pharmacology, and review its clinical evaluation in ovarian cancer to date. We focus on the favorable tolerability and encouraging signals of efficacy that have emerged, most notably in patients with platinum-resistant disease. Ongoing Phase III monotherapy and Phase Ib/II combination trials evaluating its activity in the setting of platinum resistance are emphasized, which will help define its role in the evolving landscape of ovarian cancer therapy.

First draft submitted: 1 August 2017; Accepted for publication: 21 September 2017; Published online: 3 November 2017

Keywords: antibody-drug conjugate $\bullet$ folate receptor- $\alpha \bullet$ mirvetuximab soravtansine $\bullet$ novel therapy $\bullet$ ovarian cancer $\bullet$ platinum resistance

\section{Ovarian cancer landscape}

Ovarian cancer remains a leading cause of gynecological cancer mortality, responsible for 152,000 deaths annually worldwide [1]. In the USA alone, it is estimated that 22,440 women will be diagnosed with ovarian cancer in 2017 and more than 14,000 will die due to this disease [2]. The remarkable degree of cellular and molecular heterogeneity exhibited by tumors of the ovary (including those arising from the fallopian tubes and primary peritoneum) is such that ovarian cancer is no longer considered a single disease entity with variable morphology, but rather a collection of neoplasms each associated with their own distinct histological subtypes and response to therapy [3,4]. Among these, epithelial ovarian cancer (EOC) accounts for approximately $95 \%$ of ovarian cancer malignancies [5,6]. The foundation of current standard-of-care treatment for women diagnosed with EOC is cytoreductive (debulking) surgery and chemotherapy using a platinum-based combination regimen, most commonly the carboplatin-paclitaxel doublet. This strategy has largely reached a plateau of effectiveness in improving overall patient survival $[7,8]$. The continuing high mortality is associated, at least in part, with the fact that EOC is overwhelmingly diagnosed at an advanced stage. Furthermore, while EOC is highly chemosensitive and most individuals achieve remission with front-line therapy, up to $80 \%$ of patients relapse and require further treatment without the expectation of cure [9].

The development of platinum resistance in EOC appears to be a dynamic and multifactorial process, although the underlying molecular mechanisms remain poorly defined $[10,11]$. Recurrent EOC is classified based on the length 
of time, empirically divided into 6-month blocks, since receiving treatment with a platinum agent, known as the platinum-free interval $[7,12]$. The classification is as follows:

- Refractory: progression during or within 4 weeks of platinum-based chemotherapy;

- Primary resistant: relapse within 6 months of completing initial platinum therapy;

- Partially platinum sensitive: relapse between 6 and 12 months;

- Platinum sensitive: relapse beyond 12 months.

Patients with platinum-sensitive disease have a high likelihood of responding to additional platinum-based therapy, however, almost all will eventually develop resistance, at which point they are considered to have acquired or secondary platinum resistance $[9,13]$. Both primary and acquired resistance to platinum impart a highly negative prognosis and thus there exists a substantial unmet medical need for novel and effective therapeutic approaches to improve clinical outcomes for these patients.

Although a number of therapeutic options currently exist for patients with relapsed EOC, response rates and, importantly, duration of response remain disappointingly poor. Individuals with platinum-sensitive recurrences can expect response rates of $30-90 \%$ to further platinum-based therapy $[9,14,15]$ yet only exhibit a median overall survival (OS) of 2-3 years [16]. Response rates for platinum-sensitive disease are not recommended by the Society of Gynecologic Oncology as reliable end points due to their difficulty to measure accurately and reproducibly [17]. Moreover, the outlook for refractory or primary resistant patients is particularly dismal, with response rates to subsequent lines of therapy below $20 \%$, progression-free survival (PFS) of 3-4 months and median OS less than a year [9]. In the platinum-resistant setting, treatment involves a number of nonplatinum agents used as monotherapy, including paclitaxel, docetaxel, pegylated liposomal doxorubicin (PLD), gemcitabine and topotecan [18]. None of these agents stand out as superior over others in terms of safety or efficacy. Furthermore, the clinical experience in advanced and relapsed EOC has shown that addition of a third cytotoxic compound to existing chemotherapeutic regimens results in increased toxicity without improving disease control [19-21]. Maintenance therapy with conventional cytotoxic agents has not demonstrated any meaningful OS benefit, yet cumulative toxicity associated with long-term chemotherapy has emerged as a major clinical concern [22].

More recently, an increased understanding of the biological and genomic complexity of EOC has prompted the exploration of molecularly targeted strategies designed to advance the field beyond the limitations of broadbased cytotoxic therapy $[4,8]$. In this regard, two new classes of targeted agents have been approved for EOC therapy in the last 5 years - angiogenesis inhibitors (bevacizumab) and PARP inhibitors (olaparib, rucaparib and niraparib) [23] - each of which selectively impact oncogenic pathways linked to ovarian tumorigenesis [24,25]. One strategy designed to improve patient outcomes involves combining targeted agents, which possess distinct mechanisms of action and favorable tolerability, with established chemotherapeutics [26]. Validation of this approach is exemplified by the approval of bevacizumab for use alongside paclitaxel, PLD or topotecan in platinum-resistant, recurrent disease $[27,28]$. Furthermore, the approval of bevacizumab, olaparib and niraparib as maintenance therapy underscores the degree to which targeted agents are presently impacting the treatment landscape for patients with advanced ovarian cancer.

\section{Folate receptor- $\alpha$ as a rational therapeutic target}

Folate is a B vitamin that plays essential roles in cellular metabolism as well as DNA synthesis, methylation and repair [29]. The hydrophilic nature of folate at physiological $\mathrm{pH}$ necessitates its active transport into cells, which is mediated through at least three distinct transporters [30]. The first, and major, mechanism occurs through interaction with the low-affinity reduced folate carrier, an ubiquitously expressed protein that binds reduced folate and promotes cellular uptake via a bidirectional anion-exchange mechanism [31]. Second, the proton-coupled folate transporter also facilitates bidirectional transport of folate across membranes, and appears primarily responsible for absorption of dietary folates in the intestine [32]. Third, unidirectional transport of folate into cells may occur via receptormediated endocytosis, facilitated by the high-affinity folate receptor (FR) family of cell surface transmembrane glycoproteins [33], the best characterized member of which is FR $\alpha$. This receptor shows a restricted distribution pattern in normal tissues, with expression limited to a variety of polarized epithelia, such as those found in the choroid plexus, kidney, lung and placenta [33,34]. In contrast, aberrant FR $\alpha$ overexpression is characteristic of a number of epithelial tumors, including ovarian, endometrial, triple-negative breast and non-small-cell lung cancers (NSCLC) [35]. For EOC, independent studies have reported $80-96 \%$ of tumors having constitutive expression of 
$\mathrm{FR} \alpha$, which is absent in the normal ovarian epithelium [36-39]. Moreover, these increased receptor levels have been suggested to be associated with more poorly differentiated, aggressive tumors as well as resistance to conventional chemotherapy [40,41]. Thus, FR $\alpha$ has emerged as an attractive candidate for molecularly targeted approaches designed to exploit its differential distribution pattern as a novel avenue for therapeutic intervention in EOC [42].

Initial attempts to therapeutically target the FR included investigation of farletuzumab, a humanized anti-FR $\alpha$ monoclonal antibody, which exerts antitumor activity through antibody-dependent cell-mediated cytotoxicity and complement-dependent cytotoxicity [43]. An alternative approach evaluated two small-molecule folate-cytotoxic agent conjugates, BMS-748285 and vintafolide, as a means of selectively delivering cytotoxic compounds directly to cancer cells with high FR expression [44,45]. Both farletuzumab and vintafolide (a folate-vinca alkaloid conjugate) underwent advanced clinical evaluations sufficient to provide critical proof-of-concept evidence for FR $\alpha$ as a druggable target for cancer treatment [46]. Unfortunately, each of these agents exhibited only modest singleagent activity and neither demonstrated meaningful efficacy over chemotherapy alone when evaluated as part of combination regimens in advanced-stage EOC in Phase III trials $[47,48]$. However, subset analysis of both studies showed subpopulations responding to each agent $[49,50]$.

\section{Antibody-drug conjugates}

In addition to its differential expression, the ability of FR $\alpha$ to internalize large molecules makes the receptor well suited for antibody-drug conjugate (ADC)-based therapeutic strategies. ADCs are complex engineered molecules that consist of a monoclonal antibody, directed toward tumor-associated antigens, conjugated via a stable linker to a potent cytotoxic agent. In this manner, ADCs couple the targeting and pharmacokinetic features of the antibody moiety with the additional cancer-killing impact of the cytotoxic payload [51]. Importantly, this unique method of site-selective drug delivery affords a means to reduce off-target toxicities in patients by limiting the exposure of normal tissues to the payload [52]. Clearly, appropriate antigen selection is critical for the ultimate activity and tolerability of ADC-based treatment modalities. Furthermore, all three components - antibody, linker and payload - are also essential for ADC design and optimization in order to confer maximal efficacy with minimal toxicity (reviewed in [53,54]). The development of the first generation of ADCs over a decade ago was hampered by a number of pharmacological and safety considerations. Since that time, substantial technological advancements affecting each of these aspects, along with the realization that ADCs offer a means of utilizing highly potent compounds, which on their own are too toxic to be clinically useful, have resulted in significant translational progress of this class of agents within the field of oncology. This is evidenced by the current regulatory approval of four ADCs for cancer treatment: brentuximab vedotin (a conjugate of an anti-CD30 antibody with monomethyl auristatin E), ado-trastuzumab emtansine (T-DM1, a conjugate of the anti-HER2 antibody trastuzumab with the maytansinoid compound DM1), inotuzumab ozogamicin and gemtuzumab ozogamicin (conjugates of CD22and CD33-targeting antibodies, respectively, with calicheamicin) [53]. Presently, there are more than 60 others undergoing clinical evaluation, the majority of which utilize a version of the two potent microtubule-disrupting agents found in the first two ADCs mentioned above as their payload (i.e., auristatin or maytansine derivatives) [54].

\section{Mirvetuximab soravtansine, the first FR $\alpha$-targeting ADC}

Mirvetuximab soravtansine (formerly IMGN853) is a rationally designed ADC comprised of a humanized FR $\alpha$ binding monoclonal antibody (M9346A) to which the cytotoxic maytansinoid effector molecule DM4 is attached via a cleavable disulfide linker [55,56]. High-affinity binding of the conjugate to FR $\alpha$, followed by internalization, results in intracellular accumulation of DM4 - which subsequently acts as a potent antimitotic agent through its ability to suppress microtubule dynamics [57,58]. M9346A was selected as the antibody 'backbone' by screening a panel of murine anti-FR $\alpha$ antibodies for receptor-binding affinity, followed by further optimization based on the capacity to deliver a maytansinoid payload to FR $\alpha$-positive cells using a high-throughput indirect cytotoxicity assay [59]. The antibody was then humanized by variable domain resurfacing. Evaluation of conjugates of M9346A with various linkers and maytansinoid moieties demonstrated that conjugation of DM4 with the disulfide hydrophilic linker sulfo-SPDB [ $N$-succinimydl 4-(2-pyridyldithio)-2-sulfobutanoate] provided the most potent activity in FR $\alpha$-expressing xenograft tumor models [59], and was thus incorporated into the design of the final ADC molecule. In mirvetuximab soravtansine, each antibody molecule is conjugated to an average of 3-4 molecules of DM4.

As with other ADCs, the functional activation of mirvetuximab soravtansine requires internalization and catabolism of the conjugate molecule in order to release its cytotoxic payload. A model for mirvetuximab so- 


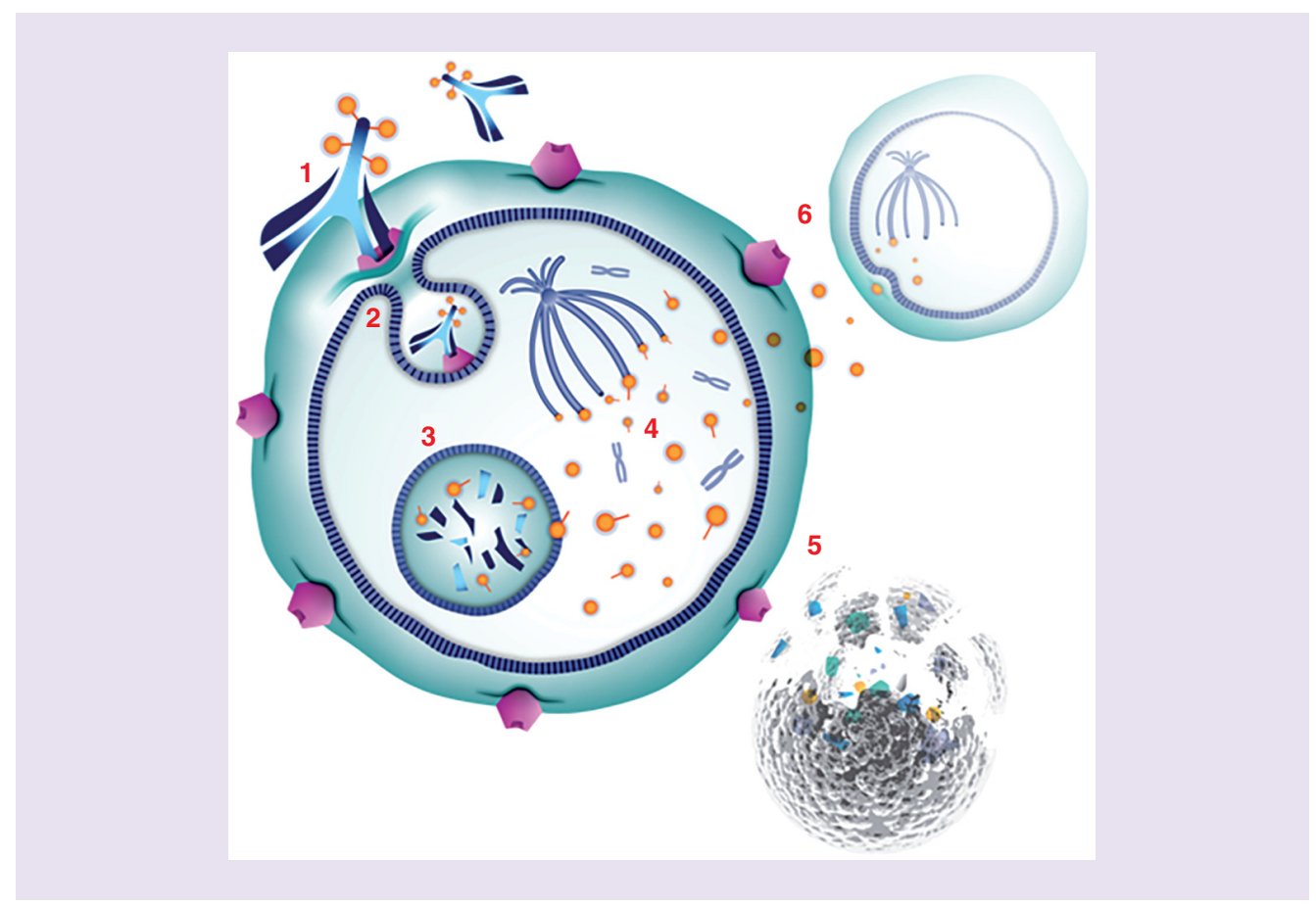

Figure 1. Mirvetuximab soravtansine processing and mechanism of action. (1) Mirvetuximab soravtansine binds with high affinity to FR $\alpha$ expressed on the tumor cell surface; (2) the antibody-drug conjugate (ADC)/receptor complex becomes internalized via antigen-mediated endocytosis; (3) lysosomal processing releases active DM4 catabolites from the ADC molecule; (4) these maytansinoid derivatives inhibit tubulin polymerization and microtubule assembly; (5) the potent antimitotic effects result in cell-cycle arrest and apoptosis; (6) active metabolites can also diffuse into neighboring cells and induce further cell death, in other words, bystander killing. Reproduced with permission from [63].

Copyright (c) 2016 Prous Science, SAU or its licensors. All rights reserved.

ravtansine processing and mechanism of action is shown in Figure 1. Mirvetuximab soravtansine is activated in a manner akin to that reported for other disulfide-linked antibody-maytansinoid conjugates [60]. Mirvetuximab soravtansine becomes internalized by tumor cells via antigen-mediated endocytosis, delivered to lysosomes by vesicular trafficking and then degraded to release lysine-NE-sulfo-SPDB-DM4. The lysine-DM4 catabolite undergoes further intracellular reduction and S-methylation, leading to the formation of the hydrophobic maytansinoid derivatives, DM4 and S-methyl-DM4. These three catabolites then exert potent antimitotic activity, via inhibition of tubulin polymerization and microtubule assembly, causing cell death. Furthermore, DM4 and S-methyl-DM4 are able to diffuse from antigen-positive tumor cells into neighboring cells and kill them in an antigen-independent manner, an effect known as 'bystander' killing [61]. Such activity is expected to be beneficial in vivo where FR $\alpha$ expression on tumor cells may be heterogeneous, and/or ADC tumor penetration could be limited. Importantly, due to the proximal nature of the bystander effect and the relative resistance of normal nondividing cells to the tubulin-disrupting action of maytansinoids, potential toxicities manifesting in normal cells due to this mechanism are likely to be minimal [62].

The preclinical characterization of mirvetuximab soravtansine demonstrated that it reduced viability in FR $\alpha$ positive tumor cells in vitro with low nanomolar potency and, confirming design expectations, surface-expressed receptor levels were identified as a key determinant of sensitivity to mirvetuximab soravtansine exposure [59]. In multiple FR $\alpha$-positive tumor models in vivo, mirvetuximab soravtansine exhibited robust single-agent activity at doses well below its maximum tolerated dose, including partial and/or complete regressions in both cell line and patient-derived xenograft models of ovarian cancer. In addition, the ADC lacked efficacy in FR $\alpha$-negative ovarian xenografts and an isotype-matched, nonbinding control conjugate was inactive in FR $\alpha$-positive models, providing further evidence of selective, antigen-directed activity [59].

Pharmacokinetic studies evaluating single intravenous (iv.) administration of mirvetuximab soravtansine in both mice and cynomolgus monkeys revealed biphasic pharmacokinetics, with an initial distribution phase lasting 
approximately $24 \mathrm{~h}$ followed by a slower terminal elimination phase [63]. In the first-in-human clinical study, mirvetuximab soravtansine was iv. dosed on day 1 of a 21 -day cycle (range: $0.15-7.0 \mathrm{mg} / \mathrm{kg}$ ). After the first administration, mean exposure (as assessed by maximum plasma concentration $\left[\mathrm{C}_{\max }\right]$ and area under concentration-time curve zero to infinity $\left[\mathrm{AUC}_{0-\infty}\right]$ ) increased in a generally dose-proportional manner across the $1.0-7.0 \mathrm{mg} / \mathrm{kg}$ range and the mean half-life values $\left(\mathrm{t}_{\frac{1}{2}}\right)$ ranged from 79 to $121 \mathrm{~h}$ across all dose levels. Importantly, the terminal half life determined at doses $\geq 1.0 \mathrm{mg} / \mathrm{kg}$ showed no dose dependence with respect to clearance or volume of distribution parameters. Cycle 3 exposure metrics indicated that there was no meaningful accumulation of mirvetuximab soravtansine after multiple doses [64].

\section{Phase I clinical experience}

Dose escalation in the first-in-human Phase I trial (NCT01609556) evaluated mirvetuximab soravtansine administered iv. on day 1 of a 21-day cycle (i.e., once every 3 weeks) to patients with FR $\alpha$-positive solid tumors, which included individuals with ovarian, endometrial, cervical, renal and NSCLC [64]. A total of 44 patients were enrolled, with the first 30 receiving mirvetuximab soravtansine at escalating doses from 0.15 to $7.0 \mathrm{mg} / \mathrm{kg}$ calculated on total body weight. Dose-limiting toxicities (DLTs) of grade 3 hypophosphatemia and grade 3 punctate keratitis were observed in one patient each at the 5.0 and $7.0 \mathrm{mg} / \mathrm{kg}$ dose levels, respectively. A modification to dosing based on adjusted ideal body weight (AIBW) was implemented in order to decrease interpatient variability in drug exposure and subsequently enrolled patients received mirvetuximab soravtansine at either 5.0 or $6.0 \mathrm{mg} / \mathrm{kg}$ AIBW ( $\mathrm{n}=7$ per group). No DLTs were observed and, based on a collective evaluation of safety, activity and PK data, dosing at $6.0 \mathrm{mg} / \mathrm{kg}$ AIBW every 3 weeks was established as the recommended Phase II dose (RP2D). Of note, this RP2D was used in a number of expansion cohorts (see below), where tolerability was confirmed.

The principal treatment-related adverse effects included fatigue and diarrhea, with the majority of cases being mild (grade 1 or 2) and readily managed without requiring discontinuation of therapy. This safety profile is similar to that reported for the Phase I evaluation of farletuzumab, but without the high incidence of hypersensitivity and infusion-related reactions [65,66]. Grade 1 or 2 peripheral neuropathy, typically associated with tubulin-targeting chemotherapy, occurred in $21 \%$ of patients and was likely a consequence of the maytansinoid payload [67]. This compares favorably with the incidence observed with conventional tubulin-disrupting cytotoxics, such as paclitaxel, particularly since a majority of these individuals had prior history of neuropathy following previous platinum/taxane therapy.

Reversible ocular adverse events (AEs), primarily manifesting as blurred vision and/or corneal keratopathy, were considered AEs of interest in the study. Grade 3 corneal opacity and punctate keratitis were reported as a serious $\mathrm{AE}$ and DLT in individuals treated at 5.0 and $7.0 \mathrm{mg} / \mathrm{kg}$ total body weight, respectively. Following the change to AIBW dosing, the visual and corneal abnormalities observed were generally mild ( $\leq$ grade 2 ) and similar in nature to those reported for other DM4-conjugated antibodies [68]. Due to the lack of FR $\alpha$ expression in the eye, it is reasonable to conclude that these effects were off-target and independent of target antigen expression. Indeed, ocular AEs have emerged as an important clinical consideration for a number of ADCs that target different antigens and employ a variety of tubulin-interacting payloads [69]. Similar ocular disorders were also observed at comparable frequencies in patients treated as part of the expansion phases of this trial. Early recognition prompted the mandating of daily lubricating eye drop use and implementation of this proactive measure, in conjunction with other ocular management procedures (e.g., avoidance of contact lenses, regular cleaning and warm compress use, sunglasses in daylight, etc.), subsequently decreased both the incidence and grade of visual disturbances in patients on study. Separately, the trial was further amended to include an expansion cohort implementing primary prophylaxis with corticosteroid eye drops to determine the effectiveness of this strategy for reducing the prevalence of ocular toxicities associated with mirvetuximab soravtansine. The findings of that expansion study are yet to be reported. A summary of the ocular management procedures implemented as part of the trial is presented in Table 1 .

With respect to antitumor activity, the strongest signals of clinical benefit were observed in the subset of patients with EOC. These included two cases of RECIST-defined partial responses (PR) as well as four additional individuals with confirmed CA 125 responses and two with stable disease lasting more than 4 months.

The development of mirvetuximab soravtansine as a highly selective, directed therapeutic agent has necessitated reliable quantification of tumor FR $\alpha$ expression in order to use this measure as a response-predictive biomarker for patient selection [37]. A variety of methods have been used to detect FR $\alpha$ expression in tumors, including efforts to develop noninvasive folate-targeting radioimaging agents [70]. Patient enrollment into mirvetuximab soravtansine trials is based on immunohistochemical assessment of archival tumor tissue, a detection method that offers the 


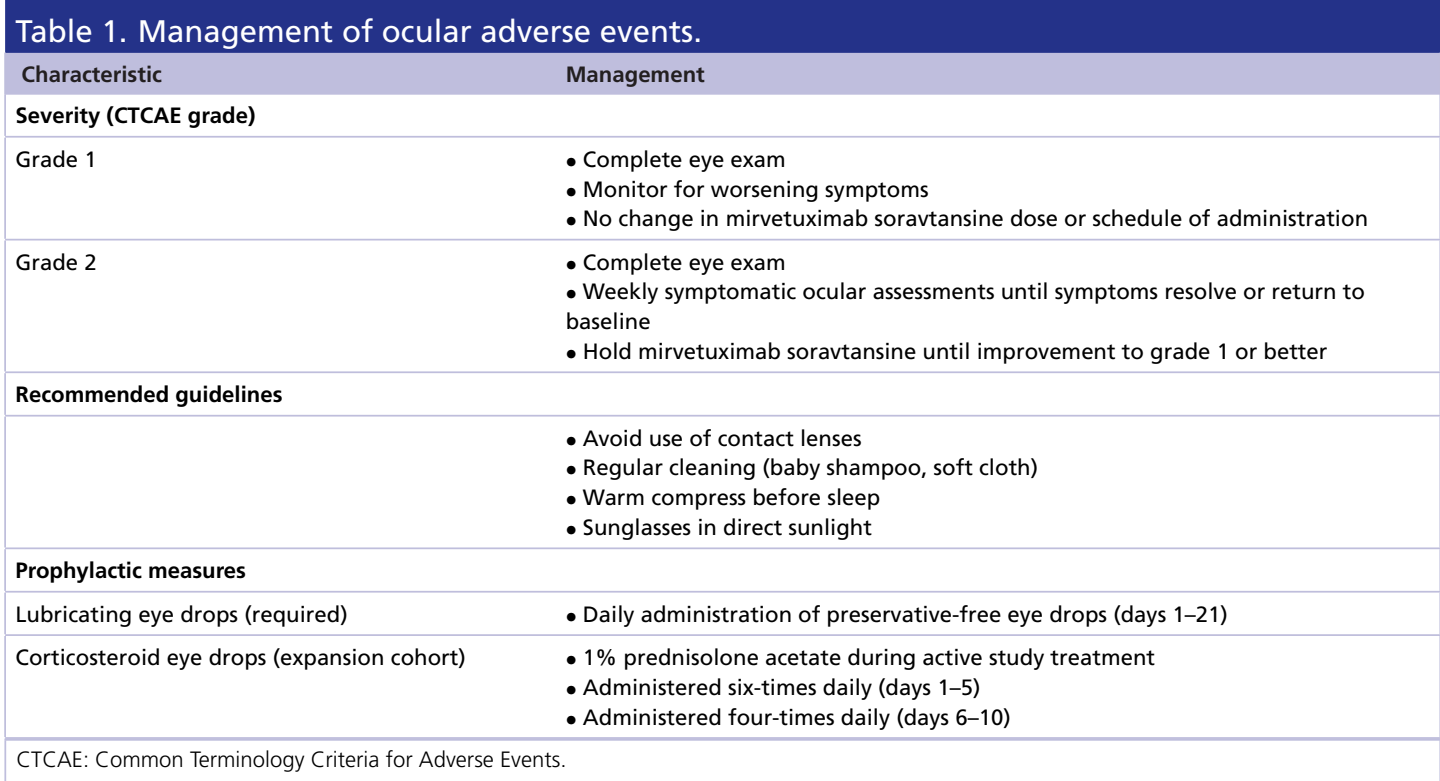

ability to evaluate uniformity and intensity of FR $\alpha$ expression with sufficient sensitivity and specificity. However, a caveat of this approach is the possibility that the level of FR $\alpha$ expression observed in archival tissue may not be representative of FR $\alpha$ expression at the time of treatment. In this regard, it has been reported that FR $\alpha$ expression is not significantly altered in response to chemotherapy [71], an observation consistent with subsequent findings in platinum-resistant ovarian cancer tissue samples [72]. Taken together, these studies support the rationale for targeting $\mathrm{FR} \alpha$ in the treatment of EOC, whether newly diagnosed or at the time of recurrence.

As part of the same Phase I trial, another expansion cohort was opened in order to compare FR $\alpha$ expression in archival tumor and fresh biopsy samples from a heterogeneous population of patients with relapsed ovarian cancer [73]. 27 patients were enrolled and treated with mirvetuximab soravtansine on the RP2D regimen. All patients were heavily pretreated (median of four prior systemic therapies, range 1-11), had previous platinum/taxane exposure and met the minimum requirement for FR $\alpha$ positivity of $\geq 25 \%$ of tumor staining at $\geq 2+$ intensity, based on assessment of archival tumor specimens. One limitation of biopsy collection uncovered in the study was that a significant proportion (22\%) of fresh samples lacked sufficient tumor tissue for evaluation, likely due to the intrinsically small sample volume provided by a core biopsy compared with the amount of tissue procured as part of archival tumor collection. Overall, the concordance of FR $\alpha$ levels in archival tissues and evaluable fresh biopsy samples $(71 \%)$ suggested that tumor receptor expression was preserved in the interval between original diagnosis and recurrence, even after multiple lines of prior therapy, and that immunohistochemical evaluation of archival tissue is appropriate for patient selection in the recurrent setting, at least when administering ADC-targeting agents.

In this heavily pretreated ovarian cancer population, confirmed tumor responses were observed in six patients, comprising two complete responses (CRs) and four PRs, for an objective response rate (ORR) of $22 \%$. As part of the analyses, tumor tissues were scored for FR $\alpha$ positivity as either low, medium or high (25-49\%, 50-74\% or $\geq 75 \%$ of cells with $\geq 2+$ staining intensity, respectively). Of note, and regardless of the tissue source analyzed (archival or biopsy), higher FR $\alpha$ expression was associated with greater antitumor activity, as evidenced by trends toward improved ORR and PFS, particularly in patients classified as high FR $\alpha$ expressers [73]. This result underscores the importance of incorporating patient stratification, based on receptor expression status, into the design of clinical trials.

\section{Mirvetuximab soravtansine is active in patients with platinum-resistant ovarian cancer}

The encouraging signals of clinical activity to emerge from the early Phase I findings with mirvetuximab soravtansine in patients with advanced EOC led to investigation of monotherapy in a defined expansion cohort of patients with platinum-resistant disease [74]. 46 patients with FR $\alpha$-positive tumors were enrolled, again based on an inclusion threshold of $\geq 25 \%$ of cells with $\geq 2+$ staining intensity. Patients with up to five prior lines of systemic therapy were eligible to enroll; 11 patients (24\%) had received only one prior platinum regimen (i.e., primary platinum 
Table 2. Confirmed objective response rates and time to event end points.

\begin{tabular}{|lll|}
\hline End point & All pooled patients $(\mathbf{n}=113)$ & FORWARD I-eligible $(\mathbf{n}=36)$ \\
\hline $\begin{array}{l}\text { Confirmed ORR } \\
95 \% \mathrm{Cl}\end{array}$ & $3 \%^{\dagger}$ & $\begin{array}{l}4 \%^{\ddagger} \\
(30,65)\end{array}$ \\
\hline PFS (months) & $(22,39)$ & \\
Median & & 6.7 \\
$95 \% \mathrm{Cl}$ & 4.3 & $(4.1,8.3)$ \\
\hline DOR (weeks) & $(3.9,5.4)$ & \\
Median & & 25.1 \\
$95 \% \mathrm{Cl}$ & 19.3 & $(18.0,42.0)$ \\
\hline
\end{tabular}

$\dagger$ Three complete response and 31 partial response.

One complete response and 16 partial response.

DOR: Duration of response; ORR: Objective response rate; PFS: Progression-free survival.

Reproduced with permission from [78].

resistance), while the remaining 35 (76\%) had received at least two lines of platinum-based therapy (i.e., acquired platinum resistance).

For the overall patient population, the confirmed ORR was $26 \%$, including $1 \mathrm{CR}$ and $11 \mathrm{PRs}$, and the median PFS was 4.8 months. These values compare favorably with chemotherapeutic agents currently used in the secondline setting for recurrent EOC. For example, in a Phase III trial directly comparing PLD and topotecan, both drugs were equally ineffective in the subset of EOC patients defined by chemoresistant disease (ORR of 12 vs $7 \%$, and a median PFS of 9.1 vs 13.6 weeks, respectively) [75]. In a separate Phase III trial in patients with recurrent EOC (who experienced treatment failure within 12 months of receipt of only one prior platinum/paclitaxel regimen), gemcitabine demonstrated an overall response rate of $29 \%$ and median time to progression of 20 weeks [76].

Of particular note, when patients were stratified based on the number of previous therapies, the subset of individuals enrolled with $1-3$ priors $(n=23)$ demonstrated a response rate of $39 \%$ and a median PFS of 6.7 months (compared with $13 \%$ and 3.9 months for patients with four or more prior lines, $n=23$ ) [74]. These efficacy measures for mirvetuximab soravtansine monotherapy are comparable to those observed in the pivotal AURELIA trial (ORR: $31 \%$ and median PFS $=6.7$ months) [77] that led to US FDA approval of bevacizumab for use alongside chemotherapy in patients with platinum-resistant EOC who have received no more than two prior therapeutic regimens.

This finding was instrumental in defining the target population for the recently initiated, pivotal study of mirvetuximab soravtansine monotherapy, FORWARD I (NCT02631876). FORWARD I is a randomized Phase III trial comparing the safety and efficacy of mirvetuximab soravtansine to physicians' choice chemotherapy in platinum-resistant EOC, fallopian tube or peritoneal cancer patients. Taking into consideration the putative relationship between the level of tumor FR $\alpha$ expression and response, patient eligibility is based on three key eligibility criteria: platinum-resistant disease, 1-3 prior lines of therapy and medium/high FR $\alpha$ expression (i.e., $\geq 50 \%$ of cells with $\geq 2+$ staining intensity). Robust support for this enrollment strategy was provided by a retrospective pooled analysis of safety and efficacy performed in individuals enrolled across three expansion cohorts from the initial Phase I trial [78]. A total of 113 patients had been treated as part of the platinum-resistant, biopsy and corticosteroid eye drop cohorts described previously; of these, 36 patients were considered the 'FORWARD I-eligible' subset, meeting the criteria listed above. Mirvetuximab soravtansine was well tolerated across the entire pooled population ( $\mathrm{n}=113$ ), with the most common AEs being diarrhea, fatigue, nausea and blurred vision. These were generally grade 1 or 2 and readily managed, and no grade $\geq 3 \mathrm{AE}$ was present in $\geq 10 \%$ of patients. Moreover, drug-related AEs leading to discontinuation were observed in only $9 \%$ of patients. The AE profile for the FORWARD I-eligible subset was consistent with the overall pooled population. Perhaps more importantly, the efficacy measures in this group were particularly encouraging, as a confirmed ORR of 47\% (1 CR and 16 PRs) and median PFS of 6.7 months was achieved (Table 2). Figure 2 shows the changes in patient target lesion burden as a function of time in this subset of patients, with individuals further stratified according to FR $\alpha$ levels. These values are superior to outcomes typically seen with established single-agent chemotherapy within the platinum-resistant disease setting. Moreover, the consistency of data across multiple cohorts underscores the therapeutic potential of mirvetuximab soravtansine for a population of cancer patients with few effective or durable treatment options. 


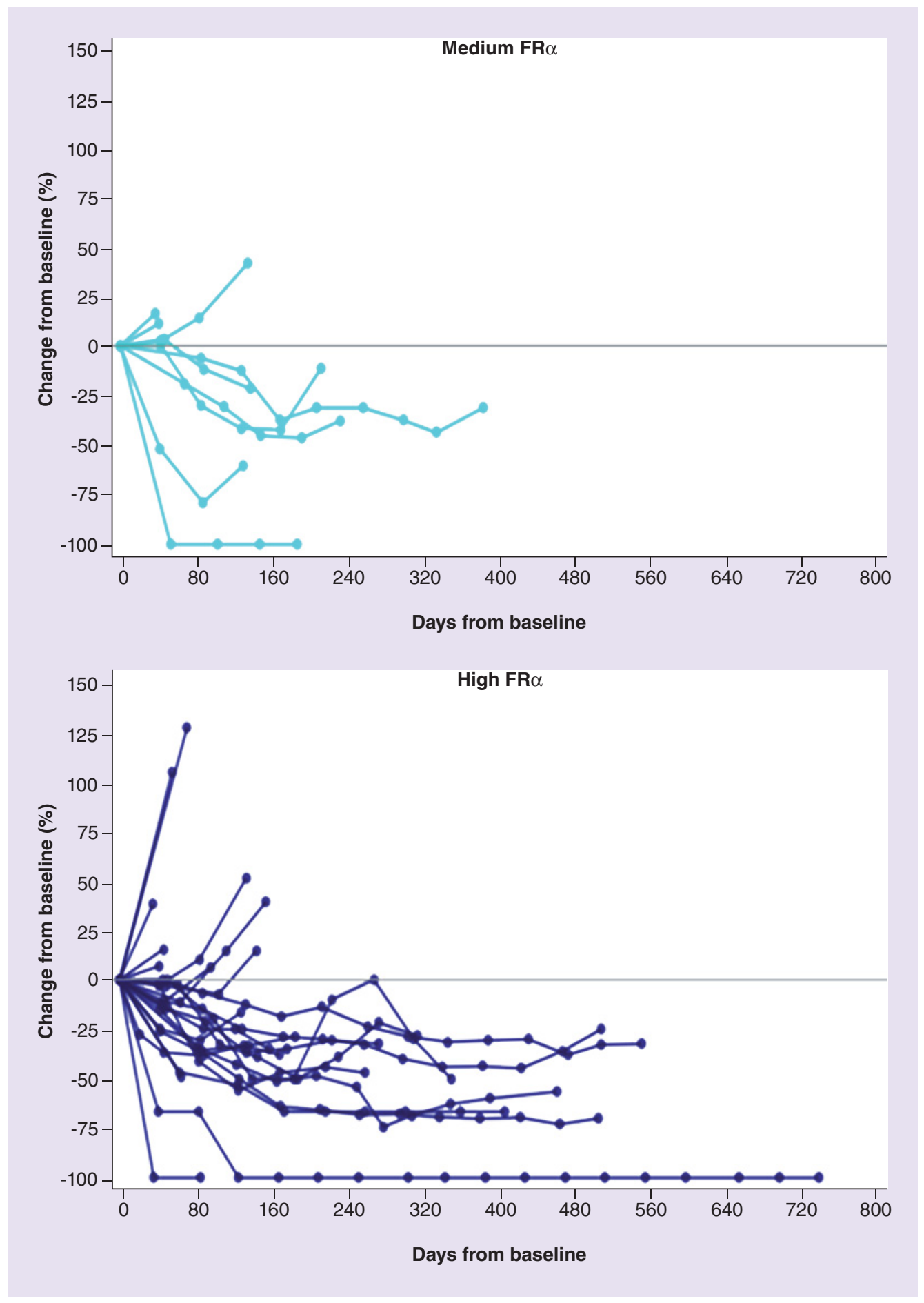

Figure 2. Percent tumor change in target lesions by FR $\alpha$ expression (FORWARD I-eligible Phase I pooled patients). Reproduced with permission from [78].

\section{Combinatorial strategies for the treatment of platinum-resistant disease}

As exemplified by the approval of bevacizumab in platinum-resistant EOC alongside established cytotoxics, the integration of molecularly targeted agents possessing alternate mechanisms of action and nonoverlapping toxicities to conventional chemotherapeutic regimens represents a promising avenue for the future management of EOC. Mirvetuximab soravtansine has been shown to potentiate the antitumor activity of established agents used in EOC 
treatment (including carboplatin, PLD and bevacizumab) in preclinical models [79]. These observations provided a framework for an ongoing Phase Ib/II study evaluating mirvetuximab soravtansine in combination with bevacizumab, carboplatin, PLD or pembrolizumab in patients with ovarian cancer (FORWARD II, NCT02606305). With the exception of the carboplatin combination, each of the regimens are being investigated in platinum-resistant EOC patient populations.

\section{Mirvetuximab soravtansine plus bevacizumab}

A significant preclinical finding was the markedly improved antitumor activity afforded by combining mirvetuximab soravtansine with bevacizumab, which caused robust and durable tumor regressions in multiple platinum-resistant EOC xenograft models. The mechanism(s) underlying this combinatorial benefit are yet to be fully elucidated. One possibility is that the presence of bevacizumab promotes better tumor penetration and exposure to the ADC, resulting in more effective eradication of tumor cells. In this regard, it is well established that bevacizumab treatment can induce normalization of the tumor vasculature, an effect suggested to result in reduced interstitial pressures and improved drug delivery [80]. However, additional preclinical and clinical data have emerged consistent with reduced tumor uptake of both chemotherapeutic drugs and antibodies following antiangiogenic therapy (reviewed in [81]). The initial findings from the dose-escalation stage of FORWARD II were presented at the ASCO Annual Meeting in 2017 [82]. Mirvetuximab soravtansine was successfully escalated to the RP2D/Phase III monotherapy dose $(6.0 \mathrm{mg} / \mathrm{kg}$ AIBW) in combination with $15 \mathrm{mg} / \mathrm{kg}$ bevacizumab; both agents were administered on day 1 of a 3 -week cycle $(n=14)$. The AE profile for the combination was manageable and expected based on the known profiles of each agent, and only one patient at the highest dosing level experienced DLTs (grade 2 neutropenia and thrombocytopenia). Moreover, dose-escalation provided encouraging signs of clinical activity in a heavily pretreated platinum-resistant population (median of six prior lines of systemic therapy, $64 \%$ of patients with previous bevacizumab exposure), with a confirmed ORR of $29 \%$ and median PFS of 9.5 months. Taken together, these early safety and efficacy findings support the planned expansion cohorts evaluating combination mirvetuximab soravtansine-bevacizumab therapy in both bevacizumab-naive and pretreated-EOC patients.

\section{Mirvetuximab soravtansine plus PLD}

In clinical practice, the anthracycline PLD is widely used as second-line monotherapy in patients with platinumresistant EOC, as well as a combination partner for bevacizumab in the same setting [83]. Preclinical data suggested that mirvetuximab soravtansine and PLD exert synergistic antitumor effects on ovarian cancer cells and, importantly, these effects translated to improved and durable efficacy over respective single-agent treatments alone (with good tolerability) in platinum-resistant, patient-derived xenografts [79]. Dose-escalation results from FORWARD II demonstrated that the combination was practical, with the highest dosing level evaluated being $6.0 \mathrm{mg} / \mathrm{kg}$ (AIBW) mirvetuximab soravtansine and $40 \mathrm{mg} / \mathrm{kg}$ PLD administered on day 1 of a 4 -week cycle $(\mathrm{n}=16)$ [82]. The safety profile of the combination was manageable, with the most frequent AEs being diarrhea (56\%), constipation (50\%), fatigue and nausea (each 44\%) and no DLTs observed.

\section{Mirvetuximab soravtansine plus pembrolizumab}

The FORWARD II trial is additionally exploring the potential for combining mirvetuximab soravtansine alongside immunotherapy in the treatment of platinum-resistant EOC. The ADC is being evaluated in combination with pembrolizumab, a humanized monoclonal antibody against PD-1. Pembrolizumab is approved for the treatment of multiple cancer indications, including advanced melanoma as well as patients with metastatic NSCLC whose tumors express PD-L1 and who have experienced disease progression on or after platinum-containing chemotherapy [84]. Immune checkpoint blockade is an active area of investigation in ovarian cancer $[85,86]$ and preliminary evidence of clinical benefit has been reported for single-agent pembrolizumab in advanced EOC patients [87]. Preclinically, mirvetuximab soravtansine has been shown to activate monocytes and upregulate immunogenic cell death markers on ovarian tumor cells - providing a mechanistic rationale for combining this agent alongside a checkpoint inhibitor [88]. The outcomes of the mirvetuximab soravtansine-pembrolizumab combination study are expected to be informative as to the feasibility of combining the two treatment modalities in this indication, particularly in light of additional preclinical findings reporting that treatment with the maytansinoid-containing ADC ado-trastuzumab emtansine can elicit antitumor immunity and render HER2 ${ }^{+}$breast tumors susceptible to immune checkpoint inhibition [89]. 


\section{Conclusion}

Primary or acquired resistance to platinum-based therapy imparts a dismal prognosis for EOC patients and effective, tolerable agents to treat this disease represent an urgent unmet medical need. FR $\alpha$ has emerged as an attractive and clinically validated candidate for the development of molecularly targeted therapeutic strategies in this indication, with mirvetuximab soravtansine representing the first FR $\alpha$-targeting ADC, purposely designed to exploit this tumor-associated antigen for site-directed drug delivery. The early clinical evaluations of mirvetuximab soravtansine have revealed favorable tolerability and encouraging activity when administered as monotherapy to patients with relapsed EOC, particularly within the setting of platinum resistance. Indeed, for less heavily pretreated individuals with higher levels of FR $\alpha$ expression (1-3 prior lines of therapy and medium/high receptor expression), the efficacy measures to date appear to be considerably better than those expected with existing standard-of-care single-agent chemotherapy in this difficult-to-treat population. Accordingly, the results of the pivotal FORWARD I trial are awaited with interest and are expected to help define the potential role of this novel agent, as well as the therapeutic utility of targeting $\mathrm{FR} \alpha$, in the management of relapsed/refractory ovarian cancer. Moreover, the exploration of mirvetuximab soravtansine in combination with established therapeutics and other investigational agents has commenced, providing an alternative therapeutic framework to achieving meaningful benefit in a disease with limited treatment options. Importantly, initial findings have shown that mirvetuximab soravtansine can be safely combined with combinatorial partners at full doses, underscoring the tolerability of this approach. Based on

\section{Executive summary}

Background

- Ovarian cancer remains a worldwide leading cause of gynecological cancer mortality, and platinum-resistant disease imparts a particularly dismal prognosis.

- Elevated FR $\alpha$ expression is characteristic of epithelial ovarian cancer (EOC), making this receptor an attractive candidate for the development of novel therapeutics in this indication.

- Earlier evaluations of FR-targeting agents provided critical proof-of-concept evidence for FR $\alpha$ as a druggable target in EOC, but lacked meaningful clinical activity.

Mechanism of action of mirvetuximab soravtansine

- Mirvetuximab soravtansine is an antibody-drug conjugate comprising a cytotoxic effector compound, the maytansinoid DM4, coupled to a humanized anti-FR $\alpha$ monoclonal antibody via a linker that is stable in the circulation but readily cleaved within cells to release the payload.

- High-affinity binding of the antibody-drug conjugate to FR $\alpha$, followed by internalization, results in high intracellular concentrations of DM4 - which subsequently acts as a potent antimitotic agent through its ability to suppress microtubule dynamics.

Phase I clinical study of mirvetuximab monotherapy

- The first-in-human, dose-escalation Phase I study of mirvetuximab soravtansine demonstrated manageable tolerability and activity in EOC patients.

- A dose of $6.0 \mathrm{mg} / \mathrm{kg}$ (adjusted ideal body weight) administered once every 3 weeks was defined as the recommended Phase II dose.

- Superior ORR and progression-free survival outcomes were observed in less-heavily pretreated patients in an expansion cohort opened to assess this regimen in patients with platinum-resistant EOC. A separate biopsy cohort confirmed a relationship between level of tumor FR $\alpha$ expression and response.

- Retrospective analyses across the Phase I trial demonstrated that platinum-resistant patients with 1-3 prior lines of therapy and medium/high FR $\alpha$ expression exhibited the strongest signals of efficacy (ORR: $47 \%$; progression-free survival, 6.7 months).

- Based on these results, the dose, schedule and target population were identified for a Phase III trial in patients with platinum-resistant disease (FORWARD I).

Combinations with other agents

- There is a preclinical rationale for combining mirvetuximab soravtansine with established therapies for EOC.

- FORWARD II is a recently initiated Phase lb study evaluating mirvetuximab soravtansine-based combinations in advanced EOC, including bevacizumab, pegylated liposomal doxorubicin and pembrolizumab within the setting of platinum resistance.

Conclusion

- The maturing clinical profile of mirvetuximab soravtansine has revealed favorable tolerability and encouraging activity in patients with platinum-resistant EOC.

- The findings to emerge from the ongoing monotherapy and combination trials will further define the role of this novel targeted agent for improving outcomes in this difficult to treat patient population. 
its maturing clinical profile, mirvetuximab soravtansine therefore appears well positioned to play an important role as part of new strategies to improve outcomes for patients with platinum-resistant ovarian cancer.

As an ADC, mirvetuximab soravtasine belongs to a clinically validated class of agents engineered to selectively deliver cytocidal amounts of chemotherapeutic agents directly to the site of tumors, in order to provide a balance between efficacy and reduced toxicity. Moreover, the feasibility of targeting FR $\alpha$ in EOC was established in previous trials of investigational agents that recognized this receptor - however, the full potential of this strategy for improving patient outcomes in ovarian and other high FR $\alpha$-expressing cancers remains to be realized. The encouraging and robust signals of efficacy in platinum-resistant EOC to emerge from the initial evaluations of mirvetuximab soravtansine suggest that the ADC holds considerable promise for providing meaningful benefit in a population with few therapeutic options beyond sequential chemotherapy. Tailored treatment strategies with combinatorial partners are an additional avenue that warrants further investigation. A successful outcome to the ongoing Phase Ib/II and Phase III studies will inform the clinical value of mirvetuximab soravtansine, which offers exciting possibilities for the future management of platinum-resistant EOC.

Financial \& competing interests disclosure

JF Ponte is an employee of ImmunoGen, Inc; KN Moore, LP Martin, DM O'Malley and MJ Birrer serve on advisory boards for ImmunoGen. The authors have no other relevant affiliations or financial involvement with any organization or entity with a financial interest in or financial conflict with the subject matter or materials discussed in the manuscript apart from those disclosed.

The authors thank R Bates, Sr Manager of Publications at ImmunoGen, for his writing and editorial assistance.

\section{References}

Papers of special note have been highlighted as: $\bullet$ of interest; $\bullet \bullet$ of considerable interest

1. Ferlay J, Soerjomataram I, Dikshit R et al. Cancer incidence and mortality worldwide: sources, methods and major patterns in GLOBOCAN 2012. Int. J. Cancer 136(5), E359-E386 (2015).

2. Siegel RL, Miller KD, Jemal A. Cancer statistics, 2017. CA Cancer J. Clin. 67(1), 7-30 (2017).

3. Cancer Genome Atlas Research N. Integrated genomic analyses of ovarian carcinoma. Nature 474(7353), 609-615 (2011).

4. Liu J, Matulonis UA. New strategies in ovarian cancer: translating the molecular complexity of ovarian cancer into treatment advances. Clin. Cancer Res. 20(20), 5150-5156 (2014).

5. Lacey JV, Sherman ME. Ovarian neoplasia. In: Robboy's Pathology of the Female Reproductive Tract. Robboy SL, Mutter GL, Prat J (Eds). Churchill Livingstone Elsevier, Oxford, UK, 601 (2009).

6. Desai A, Xu J, Aysola K et al. Epithelial ovarian cancer: an overview. World J. Transl. Med. 3(1), 1-8 (2014).

7. Luvero D, Milani A, Ledermann JA. Treatment options in recurrent ovarian cancer: latest evidence and clinical potential. Ther. Adv. Med. Oncol. 6(5), 229-239 (2014).

8. Della Pepa C, Tonini G, Pisano C et al. Ovarian cancer standard of care: are there real alternatives? Chin. J. Cancer 34(1), 17-27 (2015).

9. Davis A, Tinker AV, Friedlander M. "Platinum resistant" ovarian cancer: what is it, who to treat and how to measure benefit? Gynecol. Oncol. 133(3), 624-631 (2014).

10. Tapia G, Diaz-Padilla I. Molecular mechanisms of platinum resistance in ovarian cancer. In: Ovarian Cancer - A Clinical And Translational Update. Diaz-Padilla I (Ed.). InTech (2013).

11. Cooke SL, Brenton JD. Evolution of platinum resistance in high-grade serous ovarian cancer. Lancet Oncol. 12(12), 1169-1174 (2011).

12. Stuart GC, Kitchener H, Bacon M et al. 2010 Gynecologic Cancer InterGroup (GCIG) consensus statement on clinical trials in ovarian cancer: report from the Fourth Ovarian Cancer Consensus Conference. Int. J. Gynecol. Cancer 21(4), 750-755 (2011).

13. Markman M, Bookman MA. Second-line treatment of ovarian cancer. Oncologist 5(1), 26-35 (2000).

14. Rose PG, Fusco N, Fluellen L, Rodriguez M. Second-line therapy with paclitaxel and carboplatin for recurrent disease following first-line therapy with paclitaxel and platinum in ovarian or peritoneal carcinoma. J. Clin. Oncol. 16(4), 1494-1497 (1998).

15. Hoekstra AV, Hurteau JA, Kirschner CV, Rodriguez GC. The combination of monthly carboplatin and weekly paclitaxel is highly active for the treatment of recurrent ovarian cancer. Gynecol. Oncol. 115(3), 377-381 (2009).

16. Wagner U, Marth C, Largillier R et al. Final overall survival results of Phase III GCIG CALYPSO trial of pegylated liposomal doxorubicin and carboplatin vs paclitaxel and carboplatin in platinum-sensitive ovarian cancer patients. Br. J. Cancer 107(4), 588-591 (2012).

17. Herzog TJ, Armstrong DK, Brady MF et al. Ovarian cancer clinical trial endpoints: Society of Gynecologic Oncology white paper. Gynecol. Oncol. 132, 8-17 (2014).

18. Oronsky B, Ray CM, Spira AI, Trepel JB, Carter CA, Cottrill HM. A brief review of the management of platinum-resistant-platinum-refractory ovarian cancer. Med. Oncol. 34(6), 103 (2017). 
19. Bookman MA, Brady MF, Mcguire WP et al. Evaluation of new platinum-based treatment regimens in advanced-stage ovarian cancer: a Phase III trial of the gynecologic cancer intergroup. J. Clin. Oncol. 27(9), 1419-1425 (2009).

20. Du Bois A, Weber B, Rochon J et al. Addition of epirubicin as a third drug to carboplatin-paclitaxel in first-line treatment of advanced ovarian cancer: a prospectively randomized gynecologic cancer intergroup trial by the Arbeitsgemeinschaft Gynaekologische Onkologie Ovarian Cancer Study Group and the Groupe d'Investigateurs Nationaux pour l'Etude des Cancers Ovariens. J. Clin. Oncol. 24(7), 1127-1135 (2006).

21. Lheureux S, Karakasis K, Kohn EC, Oza AM. Ovarian cancer treatment: the end of empiricism? Cancer 121(18), 3203-3211 (2015).

22. Korkmaz T, Seber $S$, Basaran G. Review of the current role of targeted therapies as maintenance therapies in first and second line treatment of epithelial ovarian cancer; In the light of completed trials. Crit. Rev. Oncol. Hematol. 98, 180-188 (2016).

23. Symeonides S, Gourley C. Ovarian cancer molecular stratification and tumor heterogeneity: a necessity and a challenge. Front. Oncol. 5 , $229(2015)$

24. Colombo N, Conte PF, Pignata S, Raspagliesi F, Scambia G. Bevacizumab in ovarian cancer: focus on clinical data and future perspectives. Crit. Rev. Oncol. Hematol. 97, 335-348 (2016).

25. Konecny GE, Kristeleit RS. PARP inhibitors for BRCA1/2-mutated and sporadic ovarian cancer: current practice and future directions. Br. J. Cancer 115(10), 1157-1173 (2016).

26. Banerjee S, Kaye S. The role of targeted therapy in ovarian cancer. Eur. J. Cancer 47(Suppl. 3), S116-S130 (2011).

27. Aravantinos G, Pectasides D. Bevacizumab in combination with chemotherapy for the treatment of advanced ovarian cancer: a systematic review. J. Ovarian Res. 7, 57 (2014).

28. Yoshida H, Yabuno A, Fujiwara K. Critical appraisal of bevacizumab in the treatment of ovarian cancer. Drug Des. Devel. Ther. 9, 2351-2358 (2015).

29. Choi SW, Mason JB. Folate and carcinogenesis: an integrated scheme. J. Nutr. 130(2), 129-132 (2000).

30. Assaraf YG, Leamon CP, Reddy JA. The folate receptor as a rational therapeutic target for personalized cancer treatment. Drug Resist. Updat. 17(4-6), 89-95 (2014).

31. Zhao R, Diop-Bove N, Visentin M, Goldman ID. Mechanisms of membrane transport of folates into cells and across epithelia. Annu. Rev. Nutr. 31, 177-201 (2011).

32. Desmoulin SK, Hou Z, Gangjee A, Matherly LH. The human proton-coupled folate transporter: biology and therapeutic applications to cancer. Cancer Biol. Ther. 13(14), 1355-1373 (2012).

33. Elnakat H, Ratnam M. Distribution, functionality and gene regulation of folate receptor isoforms: implications in targeted therapy. Adv. Drug Deliv. Rev. 56(8), 1067-1084 (2004).

34. Salazar MD, Ratnam M. The folate receptor: what does it promise in tissue-targeted therapeutics? Cancer Metastasis Rev. 26(1), 141-152 (2007).

35. Ledermann JA, Canevari S, Thigpen T. Targeting the folate receptor: diagnostic and therapeutic approaches to personalize cancer treatments. Ann. Oncol. 26(10), 2034-2043 (2015).

36. Kalli KR, Oberg AL, Keeney GL et al. Folate receptor alpha as a tumor target in epithelial ovarian cancer. Gynecol. Oncol. 108(3), 619-626 (2008).

37. Vergote IB, Marth C, Coleman RL. Role of the folate receptor in ovarian cancer treatment: evidence, mechanism, and clinical implications. Cancer Metastasis Rev. 34(1), 41-52 (2015).

- Summarizes the role of folate receptor in ovarian cancer, and provides a rationale for targeting this receptor in the development of new therapeutic agents.

38. Markert S, Lassmann S, Gabriel B et al. Alpha-folate receptor expression in epithelial ovarian carcinoma and non-neoplastic ovarian tissue. Anticancer Res. 28(6A), 3567-3572 (2008).

39. O'shannessy DJ, Somers EB, Smale R, Fu YS. Expression of folate receptor-alpha (FRA) in gynecologic malignancies and its relationship to the tumor type. Int. J. Gynecol. Pathol. 32(3), 258-268 (2013).

40. Chen YL, Chang MC, Huang CY et al. Serous ovarian carcinoma patients with high alpha-folate receptor had reducing survival and cytotoxic chemo-response. Mol. Oncol. 6(3), 360-369 (2012).

41. Toffoli G, Russo A, Gallo A et al. Expression of folate binding protein as a prognostic factor for response to platinum-containing chemotherapy and survival in human ovarian cancer. Int. J. Cancer 79(2), 121-126 (1998).

42. Marchetti C, Palaia I, Giorgini M et al. Targeted drug delivery via folate receptors in recurrent ovarian cancer: a review. Onco. Targets Ther. 7, 1223-1236 (2014).

43. Spannuth WA, Sood AK, Coleman RL. Farletuzumab in epithelial ovarian carcinoma. Expert Opin. Biol. Ther. 10(3), 431-437 (2010).

44. Gokhale M, Thakur A, Rinaldi F. Degradation of BMS-753493, a novel epothilone folate conjugate anticancer agent. Drug Dev. Ind. Pharm. 39(9), 1315-1327 (2013).

45. Ambrosio AJ, Suzin D, Palmer EL, Penson RT. Vintafolide (EC145) for the treatment of folate-receptor-alpha positive platinum-resistant ovarian cancer. Expert Rev. Clin. Pharmacol. 7(4), 443-450 (2014). 
46. Lutz RJ. Targeting the folate receptor for the treatment of ovarian cancer. Transl. Cancer Res. 4(1), 118-126 (2015).

47. Vergote I, Armstrong D, Scambia G et al. A randomized, double-blind, placebo-controlled, Phase III study to assess efficacy and safety of weekly farletuzumab in combination with carboplatin and taxane in patients with ovarian cancer in first platinum-sensitive relapse. $J$. Clin. Oncol. 34(19), 2271-2278 (2016).

48. Merck and Endocyte announce independent DSMB recommends vintafolide proceed Phase III trial be stopped for futility following interim analysis (2014). http://investor.endocyte.com/releasedetail.cfm?releaseid=844838

49. Naumann RW, Coleman RL, Burger RA et al. PRECEDENT: a randomized Phase II trial comparing vintafolide (EC145) and pegylated liposomal doxorubicin (PLD) in combination versus PLD alone in patients with platinum-resistant ovarian cancer. J. Clin. Oncol. 31(35), 4400-4406 (2013).

50. Kline JB, Kennedy RP, Albone E et al. Tumor antigen CA125 suppresses antibody-dependent cellular cytotoxicity (ADCC) via direct antibody binding and suppressed Fc-gamma receptor engagement. Oncotarget 8(32), 52045-52060 (2017).

51. Chari RV, Miller ML, Widdison WC. Antibody-drug conjugates: an emerging concept in cancer therapy. Angew. Chem. Int. Ed. Engl. 53(15), 3796-3827 (2014).

52. Parslow AC, Parakh S, Lee FT, Gan HK, Scott AM. Antibody-drug conjugates for cancer therapy. Biomedicines 4(3), 14 (2016).

53. Thomas A, Teicher BA, Hassan R. Antibody-drug conjugates for cancer therapy. Lancet Oncol. 17(6), e254-e262 (2016).

54. Lambert JM, Morris CQ. Antibody-drug conjugates (ADCs) for personalized treatment of solid tumors: a review. Adv. Ther. 34(5), 1015-1035 (2017).

- Provides a comprehensive review of the current status of antibody-drug conjugates undergoing advanced clinical testing, with emphasis on solid tumors.

55. Lambert JM. Drug-conjugated antibodies for the treatment of cancer. Br. J. Clin. Pharmacol. 76(2), 248-262 (2013).

56. Lutz RJ. Targeting the folate receptor for the treatment of ovarian cancer. Transl. Cancer Res. 4, 118-126 (2015).

57. Oroudjev E, Lopus M, Wilson L et al. Maytansinoid-antibody conjugates induce mitotic arrest by suppressing microtubule dynamic instability. Mol. Cancer Ther. 9(10), 2700-2713 (2010).

58. Goldmacher VS, Audette CA, Guan Y et al. High-affinity accumulation of a maytansinoid in cells via weak tubulin interaction. PLoS ONE 10(2), e0117523 (2015).

59. Ab O, Whiteman KR, Bartle LM et al. IMGN853, a folate receptor-alpha (FRalpha)-targeting antibody-drug conjugate, exhibits potent targeted antitumor activity against fralpha-expressing tumors. Mol. Cancer Ther. 14(7), 1605-1613 (2015).

- Preclinical development and characterization of mirvetuximab soravtansine.

60. Erickson HK, Park PU, Widdison WC et al. Antibody-maytansinoid conjugates are activated in targeted cancer cells by lysosomal degradation and linker-dependent intracellular processing. Cancer Res. 66(8), 4426-4433 (2006).

61. Kovtun YV, Audette CA, Ye Y et al. Antibody-drug conjugates designed to eradicate tumors with homogeneous and heterogeneous expression of the target antigen. Cancer Res. 66(6), 3214-3221 (2006).

62. Drewinko B, Patchen M, Yang LY, Barlogie B. Differential killing efficacy of twenty antitumor drugs on proliferating and nonproliferating human tumor cells. Cancer Res. 41(6), 2328-2333 (1981).

63. Gunderson CC, Moore KN. Mirvetuximab soravtansine. FR $\alpha$-targeting ADC, treatment of epithelial ovarian cancer. Drugs of the Future 41(9), 539-545 (2016).

64. Moore KN, Borghaei H, O'malley DM et al. Phase 1 dose-escalation study of mirvetuximab soravtansine (IMGN853), a folate receptor alpha-targeting antibody-drug conjugate, in patients with solid tumors. Cancer 123(16), 3080-3087 (2017).

65. Konner JA, Bell-Mcguinn KM, Sabbatini P et al. Farletuzumab, a humanized monoclonal antibody against folate receptor alpha, in epithelial ovarian cancer: a Phase I study. Clin. Cancer. Res. 16(21), 5288-5295 (2010).

66. Sasaki Y, Miwa K, Yamashita K et al. A Phase I study of farletuzumab, a humanized anti-folate receptor alpha monoclonal antibody, in patients with solid tumors. Invest. New Drugs 33(2), 332-340 (2015).

67. Gomez-Roca CA, Boni V, Moreno V et al. A Phase I study of SAR566658, an anti CA6-antibody drug conjugate (ADC), in patients (Pts) with CA6-positive advanced solid tumors (STs) (NCT01156870). J. Clin. Oncol. 34(Suppl.), Abstract 2511 (2016).

68. Parslow AC, Parakh S, Lee F, Gan HK, Scott AM. Antibody-drug conjugates for cancer therapy. Biomedicines 4(3), 14 (2016).

69. Eaton JS, Miller PE, Mannis MJ, Murphy CJ. Ocular adverse events associated with antibody-drug conjugates in human clinical trials. J. Ocul. Pharmacol. Ther. 31(10), 589-604 (2015).

70. Sega EI, Low PS. Tumor detection using folate receptor-targeted imaging agents. Cancer Metastasis Rev. 27(4), 655-664 (2008).

71. Despierre E, Lambrechts S, Leunen $\mathrm{K}$ et al. Folate receptor alpha (FRA) expression remains unchanged in epithelial ovarian and endometrial cancer after chemotherapy. Gynecol. Oncol. 130(1), 192-199 (2013).

72. Rubinsak LA, Cohen C, Khanna N, Horowitz IR, Hanley KZ. Folate receptor alpha expression in platinum resistant/refractory ovarian carcinomas and primary endocervical adenocarcinomas. Appl. Immunohistochem. Mol. Morphol. doi:10.1097/PAI.0000000000000476 (2016) (Epub ahead of print). 
73. Martin LP, Konner J, Moore KN et al. Characterization of folate receptor alpha (FR $\alpha$ ) expression in archival tumor and biopsy samples in a Phase I study of mirvetuximab soravtansine, a FR $\alpha$-targeting antibody-drug conjugate (ADC), in relapsed epithelial ovarian cancer patients. Gynecol. Oncol. 145, 34 (2017).

74. Moore KN, Martin LP, O'malley DM et al. Safety and activity of mirvetuximab soravtansine (IMGN853), a folate receptor alpha-targeting antibody-drug conjugate, in platinum-resistant ovarian, fallopian tube, or primary peritoneal cancer: a Phase I expansion study. J. Clin. Oncol. 35(10), 1112-1118 (2017).

-• Data from a Phase I expansion cohort study indicating that mirvetuximab soravtansine is clinically active in patients with platinum-resistant epithelial ovarian cancer.

75. Gordon AN, Fleagle JT, Guthrie D, Parkin DE, Gore ME, Lacave AJ. Recurrent epithelial ovarian carcinoma: a randomized Phase III study of pegylated liposomal doxorubicin versus topotecan. J. Clin. Oncol. 19(14), 3312-3322 (2001).

76. Ferrandina G, Ludovisi M, Lorusso D et al. Phase III trial of gemcitabine compared with pegylated liposomal doxorubicin in progressive or recurrent ovarian cancer. J. Clin. Oncol. 26(6), 890-896 (2008).

77. Pujade-Lauraine E, Hilpert F, Weber B et al. Bevacizumab combined with chemotherapy for platinum-resistant recurrent ovarian cancer: the AURELIA open-label randomized Phase III trial. J. Clin. Oncol. 32(13), 1302-1308 (2014).

-. Results of a pivotal Phase III trial that resulted in approval of bevacizumab for the treatment of ovarian cancer.

78. Moore KN, Matulonis UA, O'malley DM et al. Mirvetuximab soravtansine (IMGN853), a folate alpha (FR $\alpha$ )-targeting antibody-drug conjugate (ADC), in platinum-resistant epithelial ovarian caner (EOC) patients (pts): Activity and safety analyses in Phase I pooled expansion cohorts. J. Clin. Oncol. 35(Suppl.), Abstract 5547 (2017).

79. Ponte JF, Ab O, Lanieri L et al. Mirvetuximab soravtansine (IMGN853), a folate receptor alpha-targeting antibody-drug conjugate, potentiates the activity of standard of care therapeutics in ovarian cancer models. Neoplasia 18(12), 775-784 (2016).

- Preclinical studies demonstrating combinatorial benefit conferred by the addition of mirvetuximab soravtansine to established therapeutic agents used in the treatment of epithelial ovarian cancer.

80. Jain RK. Normalization of tumor vasculature: an emerging concept in antiangiogenic therapy. Science 307(5706), 58-62 (2005).

81. Arjaans M, Schroder CP, Oosting SF, Dafni U, Kleibeuker JE, De Vries EG. VEGF pathway targeting agents, vessel normalization and tumor drug uptake: from bench to bedside. Oncotarget 7(16), 21247-21258 (2016).

82. O'malley DM, Moore KN, Vergote I et al. Safety findings from FORWARD II: a Phase Ib study evaluating the folate recepotr alpha $(\mathrm{FR} \alpha)$-targeting antibody-drug conjugate (ADC) mirvetuximab soravtansine (IMGN853) in combination with bevacizumab, carboplatin, pegylated liposomal doxorubicin (PLD), or pembrolizumab in patients (pts) with ovarian cancer. J. Clin. Oncol. 35(Suppl.), Abstract 5553 (2017).

83. Ye H, Karim AA, Loh XJ. Current treatment options and drug delivery systems as potential therapeutic agents for ovarian cancer: a review. Mater. Sci. Eng. C Mater. Biol. Appl. 45, 609-619 (2014).

84. Garon EB, Rizvi NA, Hui R et al. Pembrolizumab for the treatment of non-small-cell lung cancer. N. Engl. J. Med. 372(21), 2018-2028 (2015).

85. Weiss L, Huemer F, Mlineritsch B, Greil R. Immune checkpoint blockade in ovarian cancer. Memo 9, 82-84 (2016).

86. Mittica G, Genta S, Aglietta M, Valabrega G. Immune checkpoint inhibitors: a new opportunity in the treatment of ovarian cancer? Int. J. Mol. Sci. 17(7), pii:E1169 (2016).

87. Varga A, Piha-Paul SA, Ott PA et al. Pembrolizumab in patients (pts) with PD-L1-positive (PD-L1+) advanced ovarian cancer: updated analysis of KEYNOTE-028. J. Clin. Oncol. 35(15 Suppl.), 5513-5513 (2017).

88. Skaletskaya A, Ponte JF, Chittenden T, Setiady J. Treatment of tumor cells with mirvetuximab soravtansine, a FR $\alpha$-targeting antibody-drug conjugate (ADC), activates monocytes through Fc-FcgR interaction and immunogenic cell death. J. ImmunoTher Cancer 4(Suppl. 1), 73 (2016).

89. Muller P, Kreuzaler M, Khan T et al. Trastuzumab emtansine (T-DM1) renders HER2+ breast cancer highly susceptible to CTLA-4/PD-1 blockade. Sci. Transl. Med. 7(315), 315 ra188 (2015). 